\title{
Retrospective validation of the postnatal Growth and Retinopathy of Prematurity (G-ROP) criteria in a Swiss cohort
}

\author{
Nithursa Vinayahalingam ${ }^{1}$, Jane McDougall ${ }^{2}$, Olaf Ahrens ${ }^{2}$ and Andreas Ebneter ${ }^{1 *}$
}

\begin{abstract}
Background: Currently used screening criteria for retinopathy of prematurity (ROP) show high sensitivity for predicting treatment-requiring ROP but low specificity; over $90 \%$ of examined infants do not develop ROP that requires treatment (type 1 ROP). A novel weight gain-based prediction model was developed by the G-ROP study group to increase the specificity of the screening criteria and keep the number of ophthalmic examinations as low as possible. This retrospective cohort study aimed to externally validate the G-ROP screening criteria in a Swiss cohort.
\end{abstract}

Methods: Data from 645 preterm infants in ROP screening at Inselspital Bern between January 2015 and December 2019 were retrospectively retrieved from the screening log and analysed. The G-ROP screening criteria, consisting of 6 trigger parameters, were applied in infants with complete data. To determine the performance of the G-ROP prediction model for treatment-requiring ROP, sensitivity and specificity were calculated.

Results: Complete data were available for 322 infants who were included in the analysis. None of the excluded infants had developed type 1 ROP. By applying the 6 criteria in the G-ROP model, 214 infants were flagged to undergo screening: among these, 14 developed type 1 ROP, 9 developed type 2 ROP, and 43 developed milder stages of ROP. The sensitivity for predicting treatment-requiring ROP was 100\% (Cl, 0.79-1.00), and the specificity was $41 \%$ $(\mathrm{Cl}, 0.35-0.47)$. Implementing the novel G-ROP screening criteria would reduce the number of infants entering ROP screening by approximately one third.

Conclusions: The overall prevalence of treatment-requiring ROP was low (2.15\%). Previously published performance parameters for the G-ROP algorithm were reproducible in this Swiss cohort. Importantly, all treatment-requiring infants were correctly identified. By using these novel criteria, the burden of screening examinations could be significantly reduced.

Keywords: Screening, Retinopathy of Prematurity, G-ROP, Swiss Cohort

\section{Background}

Retinopathy of prematurity (ROP) is one of the leading preventable causes of childhood vision impairment in high income countries [1]. To prevent unfavourable outcomes of ROP, all infants at our institution

*Correspondence: andreas.ebneter@med.unibe.ch

${ }^{1}$ Department of Ophthalmology, Inselspital, Bern University Hospital, University of Bern, Bern, Switzerland

Full list of author information is available at the end of the article with birthweight $(\mathrm{BW})<1500 \mathrm{~g}$ and gestational age $(\mathrm{GA})<32$ weeks undergo repeated ophthalmic examinations according to the UK retinopathy of prematurity screening guidelines [2]. Even though many extremely preterm infants develop some degree of ROP, most of them do not require any treatment. In fact, less than $10 \%$ of examined infants with the current screening criteria develop a treatment requiring ROP (type 1 ROP). Therefore, the vast majority of ophthalmic examinations are 
performed in children who never need an intervention [3].

To minimize the burden of screening, alternative criteria have been proposed by several groups [3-9]. One of the most promising algorithms, the G-ROP model, was developed using a large database $[10,11]$. It is based on the assessment of 6 trigger criteria: gestational age (GA) less than 28 weeks, birth weight (BW) less than $1051 \mathrm{~g}$, weight gain less than $120 \mathrm{~g}$ during 10 to 19 days postnatal age (PNA), weight gain less than $180 \mathrm{~g}$ during 20 to 29 days PNA, weight gain less than $170 \mathrm{~g}$ during 30 to 39 days PNA, or hydrocephalus. The G-ROP model has been validated in several populations, [12-14] which is crucial since characteristics of premature infants vary depending on their socioeconomic environment [15].

To validate the novel G-ROP prediction model externally in a Swiss cohort and to determine the performance, the G-ROP screening criteria were retrospectively tested in a representative local cohort.

\section{Methods}

This retrospective observational study was approved by the local ethics committee Kantonale Ethikkommission Bern (KEK 2020-01064) and was performed in accordance with the Declaration of Helsinki and relevant guidelines and regulations. Written informed consent was waived by the ethics committee. The data were stored in deidentified format in a REDCap ${ }^{\circledR}$ database hosted by the Clinical Trials Unit of the Faculty of Medicine of the University of Bern and the Inselspital, Bern University Hospital.

All data were retrospectively retrieved at Inselspital Bern University Hospital, which is one of the eight Swiss hospitals with level 3 neonatal intensive care. In the catchment area, the prevailing ethnicity is Caucasian, and approximately 14,500 children are born per year. On average, 168 newborns below 32 weeks GA or below $1501 \mathrm{~g} \mathrm{BW}$ are hospitalized in this unit [16].

Data from preterm infants (GA $<37$ weeks) who underwent retinal screening examinations for ROP between January 2015 and December 2019 were collected and analysed. As per the local hospital policy, the UK screening guidelines are followed to determine the need for newborns to enter ROP screening [2]. Moreover, preterm infants with $\mathrm{BW}<2000 \mathrm{~g}$ and any type of oxygen supplementation additionally entered screening according to local guidelines. After uneventful clinical course and discharge from our tertiary institution, all infants were regularly followed up in peripheral hospitals or private practices according to UK guidelines, if needed [2].

ROP outcomes were retrospectively retrieved from the neonatal medical records, along with the sequential weight measurements, infants' GA, BW, sex, and hydrocephalus status. ROP outcomes, representing the most advanced stage reached in the course of the disease, were classified into type 1 ROP, type 2 ROP, and lowgrade ROP. Low-grade ROP was defined by mild retinal changes outside the range of normal development but not severe enough to meet criteria for type 2 ROP. Data from infants with an incomplete dataset were excluded from the final analysis. Importantly, none of the infants with missing data developed type 1 ROP.

For the infants with a complete data set, the six criteria of the G-ROP prediction model (gestational age (GA) less than 28 weeks, birth weight (BW) less than $1051 \mathrm{~g}$, weight gain less than $120 \mathrm{~g}$ during 10 to 19 days PNA, weight gain less than $180 \mathrm{~g}$ during 20 to 29 days PNA, weight gain less than $170 \mathrm{~g}$ during 30 to 39 days PNA or hydrocephalus) were applied. As soon as any of these parameters are met in an infant, it qualifies for ROP screening according to this algorithm. When an infant does not meet any of the 6 criteria, retinal ROP examinations are not warranted according to the G-ROP model [11].

The performance of the G-ROP prediction paradigm was determined by calculating the sensitivity and specificity for predicting type 1 ROP. Additionally, 95\% confidence intervals were calculated by using the Wilson score method.

\section{Results}

In total, 645 preterm infants who underwent retinal examinations between 30-37 PMA were identified. For 322 of them, the complete dataset was available, and they were included in the final analysis. Seventy infants developed ROP: 14 developed ROP type 1, 9 developed ROP type 2, and 47 developed low-grade ROP (Fig. 1). The characteristics of these children are summarized in Table 1. The median BW in this cohort was $1050 \mathrm{~g}$, and the median GA was 28.4 weeks. A total of 323 infants with incomplete information about weight gain were excluded from the study. Among the excluded infants, the median BW was $1490 \mathrm{~g}$, and the median GA was 32.3 weeks. Nine excluded children developed low-grade ROP not requiring an intervention. Of note, none of them developed type 1 ROP.

By applying the G-ROP model, an alarm was triggered in 215 children (Table 2). All infants with treatment requiring ROP were identified by the two parameters $\mathrm{BW}<1051 \mathrm{~g}$ and/or GA $<28$ weeks. Thus, the G-ROP model raised alarms correctly for all 14 ROP type 1 children, all 9 children with ROP type 2, and 43 of 47 children with low-grade ROP. The 4 children who were not flagged by the G-ROP algorithm developed low-grade ROP only. All of them had additional risk factors for 


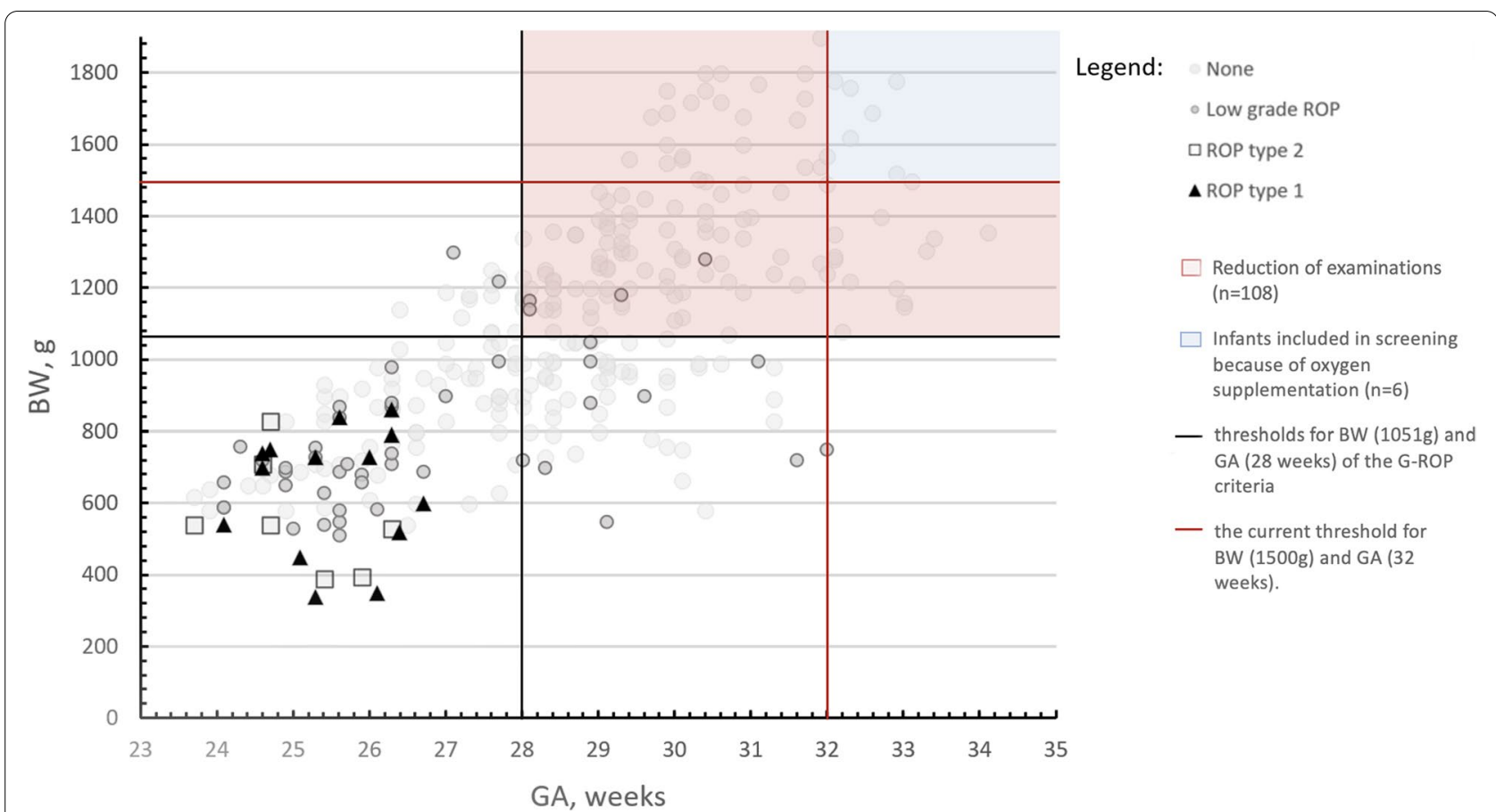

Fig. 1 Scatter plot illustrating the effect of the G-ROP criteria on screening indication. Each study subject is represented by its gestational age (GA) and birth weight (BW) in this graph. The currently used thresholds for ROP screening inclusion are indicated by the red lines. The thresholds for GA and BW in the G-ROP criteria are shown by the black lines. All infants in the area hatched in red would not have qualified for ROP screening using the G-ROP criteria. As per local guidelines, all preterm infants ( $G A<37$ weeks) with oxygen supplementation also enter ROP screening regardless of BW. These infants $(n=6)$ are represented in the upper right corner of the graph in the light blue hatched area. None of these children developed ROP. The clinical outcome related to ROP is represented by the shape of the individual data point. Infants represented by black triangles developed type 1 ROP, those represented by squares type 2 ROP, and those represented by dark circles low-grade ROP. Infants shown by the light circles did not have clinical evidence for any ROP in serial fundus examinations

Table 1 Characteristic of Infants

\begin{tabular}{|c|c|c|c|c|c|}
\hline & ROP type 1 & ROP type 2 & Low-grade & None & Excluded \\
\hline \multicolumn{6}{|l|}{ Birthweight, $\mathbf{g}$} \\
\hline mean (SD) & $637(167)$ & $596(145)$ & 799 (209) & $1134(301)$ & $1588(498)$ \\
\hline median & 705 & 540 & 720 & 1145 & 1490 \\
\hline \multicolumn{6}{|c|}{ gestational age, weeks } \\
\hline mean (SD) & $25.5(0.8)$ & $25.1(0.8)$ & $26.8(2.0)$ & $28.9(2.1)$ & $32.5(2.6)$ \\
\hline median & 25.4 & 24.7 & 26.3 & 29 & 32.3 \\
\hline male, $\mathrm{n}(\%)$ & $7(50)$ & $4(44.4)$ & 25 (53.2) & 136(53.9) & $186(53.0)$ \\
\hline
\end{tabular}

Table 2 Prediction of ROP by the G-ROP screening criteria and characteristics of infants

\begin{tabular}{llllll}
\hline & Alarm + & Alarm - & Total & Sensitivity & Specificity \\
\hline ROP type 1, n & 14 & 0 & 14 & $100 \%$ & \\
ROP type 2, n & 9 & 0 & 9 & $100 \%$ & \\
Low-grade, $\mathbf{n}$ & 43 & 4 & 47 & $91.5 \%$ & \\
None, $\mathbf{n}$ & 148 & 104 & 252 & & $41.3 \%$ \\
Total, $\mathbf{n}$ & 214 & 108 & 322 & & \\
\hline
\end{tabular}

ROP: necrotising enterocolitis, patent ductus arteriosus and prolonged oxygen supplementation with or without the diagnosis of bronchopulmonary dysplasia [11].

The sensitivity of the G-ROP criteria for predicting treatment requiring ROP was $100 \%$ (CI, 0.79-1.00), and the specificity was $41 \%$ (CI, $0.35-0.47$ ) in this cohort. Consequently, 108 infants less would have entered screening with the G-ROP algorithm, compared to currently used screening criteria [2] four of them eventually 
developed low-grade ROP, but none of them type 1 or type 2 ROP. This suggests that in this cohort, the G-ROP algorithm would have safely reduced the indication for ROP screening by $33 \%$.

\section{Discussion}

All infants with type 1 and type 2 ROP were correctly identified by the G-ROP model. Four children with lowgrade ROP were not flagged by the G-ROP algorithm and would not have entered screening. Interestingly, according to medical records, these infants all showed additional risk factors, which are associated with ROP. Nevertheless, the sensitivity for predicting treatment requiring ROP was $100 \%$, and the sensitivity for lowgrade ROP was $91 \%$. Implementing the new G-ROP screening criteria in daily clinical practice would reduce the number of screened infants by approximately one third. Similar results were found in other validation studies for the G-ROP algorithm [12-14].

Larsen et al. have suggested that modifying the GA threshold to $<30$ weeks in the screening guidelines would lower the burden of screening examinations with acceptable impact on the sensitivity of screening programs [3]. Indeed, applying this modified GA limit of $<30$ weeks would not have altered the screening sensitivity for predicting treatment-required ROP in this cohort. These findings indicate that the current screening guidelines could be re-evaluated and potentially be optimized. However, even in highly developed healthcare systems, increasing the threshold for entering ROP screening carries the risk of failure to detect infants who require sightsaving interventions.

There are some limitations to consider. Some of them are not limited to this study, but more generally apply to ROP screening criteria in any clinical settings: The influence of the socioeconomic context does not allow generalization of findings. While in some high-income countries the sensitivity of G-ROP criteria to predict type 1 ROP in validation studies was $100 \%,[11,12]$ lower sensitivity (91.9\%) has been suggested in less mature healthcare systems such as Turkey [13]. These differences may have resulted from different neonatal care practices and different characteristics of the premature population in low- and middle-income countries [15].In Switzerland and Germany, less than 1\% of infants with GA > 30 weeks developed treatment requiring ROP, $[3,17]$ but in Mexico, over one-third of preterm infants with GA $>32$ weeks may develop ROP type 1 [18]. Moreover, careful attention is needed in countries with less tightly controlled use of oxygen supplementation, as postnatal weight gain is not a reliable predictive factor of ROP when an infant is treated with excessive oxygen supplementation [14]. It is therefore extremely important to validate any new criteria in a population before adopting them.

Limitations specific to this study are the retrospective design and the single centre setup. Moreover, since data were recorded at a tertiary healthcare centre with a policy to transfer patients to peripheral hospitals close to their home as timely as medically possible, many infants had incomplete data and were excluded from the final analysis. However, according to a post-hoc sensitivity analysis, the sensitivity for predicting type 1 ROP was the same including all 645 children. In the present setting, the likelihood of missing treatment requiring disease is very low, since Inselspital Bern University Hospital is the only centre in our region that provides treatment for ROP.

\section{Conclusions}

In conclusion, the overall prevalence of treatment requiring ROP was low (2.15\%). Previously reported performance parameters for the G-ROP algorithm were reproducible in this Swiss cohort. Importantly, all treatment requiring infants were correctly identified. By using these novel criteria, the number of infants entering ROP screening could be reduced by approximately one third. These findings suggest that the current screening guidelines could be optimised, and that the G-ROP algorithm seems to be a suitable starting point to be evaluated in a larger population.

\section{Acknowledgements}

The authors would like to thank Sheila Appadoo for setting up the REDCap ${ }^{\circledR}$ database.

\section{Authors' contributions}

NV collected, analysed and interpreted the patient data, wrote the draft manuscript, and obtained ethics approval for this manuscript. JMD supervised data collection and helped with interpretation of data. OA facilitated data collection by writing scripts for extraction from medical records and contributed to the interpretation of data. AE established the conception of the study, supervised data collection, drafted the data registry and was a major contributor in writing the manuscript. All authors read and approved submission of the final manuscript and have agreed both to be personally accountable for the author's own contributions and to ensure that questions related to the accuracy or integrity of any part of the work, even those in which the author was not personally involved, are appropriately investigated, resolved, and the resolution documented in the literature.

\section{Funding}

None.

\section{Availability of data and materials}

The datasets generated and/or analyzed during the current study are not publicly available due to privacy concerns but are available from the corresponding author on reasonable request.

\section{Declarations}

Ethics approval and consent to participate

This retrospective observational study was approved by the local ethics committee Kantonale Ethikkommission Bern (KEK 2020-01064). The need for informed consent was waived because of deidentified data handling and the retrospective nature of the study. 


\section{Consent for publication \\ Not applicable}

\section{Competing interests}

Andreas Ebneter is currently an employee of F. Hoffmann-La Roche Ltd, Basel, Switzerland.

\section{Author details}

${ }^{1}$ Department of Ophthalmology, Inselspital, Bern University Hospital, University of Bern, Bern, Switzerland. ${ }^{2}$ Department of Neonatalogy, Inselspital, Bern University Hospital, University of Bern, Bern, Switzerland.

Received: 23 July 2021 Accepted: 20 December 2021

Published online: 10 January 2022

\section{References:}

1. Solebo AL, Teoh L, Rahi J. Epidemiology of blindness in children. Arch Dis Child. 2017;102(9):853-7.

2. UK Retinopathy of Prematurity Guideline. Royal College of Paediatrics and Child Health, Royal College of Ophthalmologists, British Association of Perinatal Medicine \& BLISS, London. 2008. https://www.rcophth.ac.uk/ resources-listing/uk-retinopathy-of-prematurity-guideline/. Accessed 26 Nov 2021.

3. Larsen PP, Müller A, Lagrèze WA, Holz FG, Stahl A, Krohne TU. Incidence of retinopathy of prematurity in Germany: evaluation of current screening criteria. Archives Dis Child Fetal Neonatal Ed. 2021;106(2):189-93.

4. Wirth M, Naud A, Caputo G, Hasocoet JM. The algorithm for predicting threshold retinopathy of prematurity is insufficient, and fundus examinations are still needed before 31 weeks. Acta Paediatr. 2019;108(6):1049-54.

5. McCourt EA, Ying GS, Lynch AM, Palestine AG, Wagner BD, Wymore E, et al. Validation of the Colorado Retinopathy of Prematurity Screening Model. JAMA Ophthalmol. 2018;136(4):409-16.

6. Binenbaum G, Ying GS, Quinn GE, Huang J, Dreiseitl S, Antigua J, et al. The CHOP postnatal weight gain, birth weight, and gestational age retinopathy of prematurity risk model. Arch Ophthalmol. 2012;130(12):1560-5.

7. Hellström A, Hård AL, Engström E, Niklasson A, Andersson E, Smith L, et al. Early weight gain predicts retinopathy in preterm infants: new, simple, efficient approach to screening. Pediatrics. 2009;123(4):e638-45.

8. Eckert GU, Fortes Filho JB, Maia M, Procianoy RS. A predictive score for retinopathy of prematurity in very low birth weight preterm infants. Eye. 2012;26(3):400-6.

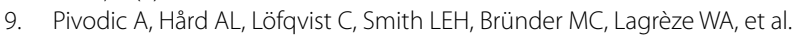
Individual risk prediction for sight-threatening retinopathy of prematurity using birth characteristics. JAMA Ophthalmol. 2020;138(1):21-9.

10. Binenbaum G, Tomlinson LA. Postnatal growth and retinopathy of prematurity study: rationale, design, and subject characteristics. Ophthalmic Epidemiol. 2017;24(1):36-47.

11. Binenbaum G, Bell EF, Donohue P, Quinn G, Shaffer J, Tomlinson LA, et al. Development of modified screening criteria for retinopathy of prematurity: primary results from the postnatal growth and retinopathy of prematurity study. JAMA Ophthalmol. 2018;136(9):1034-40.

12. Shiraki A, Fukushima Y, Kawasaki R, Sakaguchi H, Mitsuhashi M, Ineyama $H$, et al. Retrospective validation of the postnatal Growth and Retinopathy of Prematurity (G-ROP) criteria in a Japanese cohort. Am J Ophthalmol. 2019;205:50-3.

13. Yabas Kiziloglu O, Coskun Y, Akman I. Assessment of the G-ROP study criteria for predicting retinopathy of prematurity: results from a tertiary centre in Turkey. Int Ophthalmol. 2020;40(7):1647-52.

14. Binenbaum G, Tomlinson LA, de Alba Campomanes AG, Bell EF, Donohue $P$, Morrison D, et al. Validation of the postnatal growth and retinopathy of prematurity screening criteria. JAMA Ophthalmol. 2020;138(1):31-7.

15. Gilbert C, Fielder A, Gordillo L, Quinn G, Semiglia R, Visintin P, et al. Characteristics of infants with severe retinopathy of prematurity in countries with low, moderate, and high levels of development: Implications for screening programs. Pediatrics. 2005;115(5):e518-25.

16. Accredited neonatology units. Swiss Society of Neonatology, Switzerland 2021. https://www.neonet.ch/unit-accreditation/accredited-neonatology-units. Accessed 26 Nov 2021.
17. Gerull R, Brauer V, Bassler D, Laubscher B, Pfister RE, Nelle M, et al. Prediction of ROP treatment \& evaluation of screening criteria in VLBW infants-a population based analysis. Pediatr Res. 2018;84(5):632-8.

18. Zepeda-Romero LC, Hård AL, Gomez-Ruiz LM, Gutierrez-Padilla JA, Angulo-Castellanos E, Barrera-de-Leon JC, et al. Prediction of retinopathy of prematurity using the screening algorithm WINROP in a Mexican population of preterm infants. Arch Ophthalmol. 2012;130(6):720-3.

\section{Publisher's Note}

Springer Nature remains neutral with regard to jurisdictional claims in published maps and institutional affiliations.

Ready to submit your research? Choose BMC and benefit from

- fast, convenient online submission

- thorough peer review by experienced researchers in your field

- rapid publication on acceptance

- support for research data, including large and complex data types

- gold Open Access which fosters wider collaboration and increased citations

- maximum visibility for your research: over $100 \mathrm{M}$ website views per year

At BMC, research is always in progress.

Learn more biomedcentral.com/submissions 